\title{
Cerebral Mycotic Aneurysm Complicating Infectious Endocarditis Case Report and Review of the Literature
}

\author{
F. Z. Abakka ${ }^{1 *}$, I. Zouita ${ }^{1}$, S. Tadsaoui ${ }^{1}$, D. Basraoui ${ }^{1}$, H. Jalal ${ }^{1}$
}

${ }^{1}$ Radiology Mother Child Department, CHU Mohammed VI, University Cadi Ayad, Marrakech, Morocco

*Corresponding author: F. Z. Abakka

Abstract

Mycotic aneurysm is a redoubtable complication of infectious endocarditis which is the source of many other complications and is generally difficult to diagnose. They are secondary to the migration vegetations in the cerebral circulation, which, depending on its septic nature or not, can give different clinical pictures: ischemic embolic vascular accidents, meningitis, cerebral hemorrhages and brain abscesses. We report in this article a case of a 2-year-old child admitted to a picture of sudden hemiplegia evolving in a febrile context and in whom radiological exploration objectified ischemic strokes of different ages with individualization of a sacciform aneurysm of the third portion (M3) of the right sylvic artery suggesting a cerebral mycotic aneurysm complicating an infectious endocarditis which was confirmed by transthoracic ultrasound.

Keywords: Mycotic anevrysm, imaging, endocarditis.

Copyright $(\odot 2021$ The Author(s): This is an open-access article distributed under the terms of the Creative Commons Attribution 4.0 International License (CC BY-NC 4.0) which permits unrestricted use, distribution, and reproduction in any medium for non-commercial use provided the original author and source are credited.

\section{INTRODUCTION}

Cerebral mycotic aneurysms are a rare and serious complication of infectious endocarditis which engage the vital and functional prognosis. They represent less than $10 \%$ of the neurological complications of infectious endocarditis. These aneurysms result from septic embolism of vegetation in the cerebral circulation. The septic embolus, localized in the vascular lumen or at the level of the vasa vasorum, creates a suppurative inflammatory lesion of the vascular wall at the level of the intima, which diffuses by contiguity, in and out.

\section{CASE RePORT}

We report the case of a 2-year-old child, without any particular pathological priors, who has presented for 2 days with right hemiplegia and impaired consciousness developing in context of fever evaluated at $39^{\circ}$.

Clinical examination found a patient with decreased muscle strength and a positive Babinski on the right side with a mitral murmur on cardiac auscultation.

In this context of febrile consciousness impairment, a cerebral CT scan (Fig-1) was performed urgently, which was in favor of a recent ischemic stroke of the territory of the left sylvian artery associated with a sequelartriangular hypodensity under the right parietal cortex with evidence of a sacciform aneurysm of the fourth portion (M4) of the right sylvian artery. 

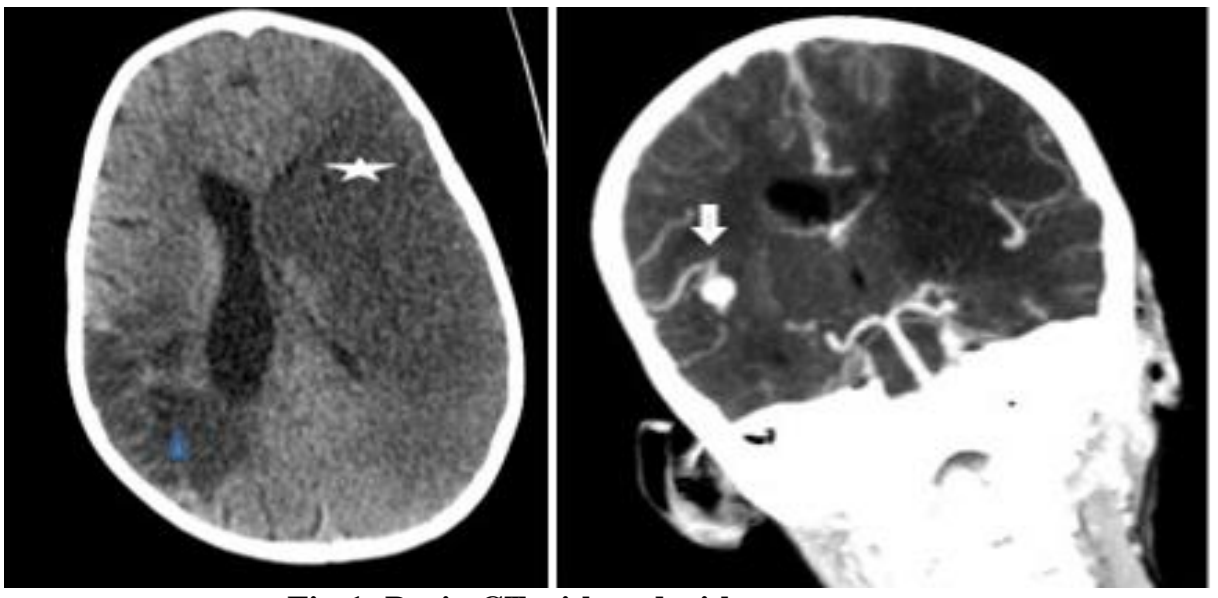

Fig-1: Brain CT with and without contrast:

Recent left parietotemporal ischemic stroke systematized in the area of the sylvian artery. $\triangle$ Old ischemic stroke of the right posterior junctional territory, site of hemorrhagic infarction. $\checkmark$ Sacciform dilation of the fourth portion (M4) of the right sylvian artery.

In light of those CT scan results, a mycotic cerebral aneurysm was suspected.

To support the diagnosis, a transthoracic ultrasound was performed objectifying the presence of large endocardial vegetation on the mitral valve indicating infectious endocarditis.

The patient received Gentamicin-based treatment with third-generation cephalosporin and antipyretic with good clinical improvement.

A few days later, for a better characterization, the child underwent a brain MRI (Fig-2) compatible with an old ischemic stroke of the right posterior junctional territory with evidence of an aneurysmal dilation of the right M4 of mycotic origin. This was associated with a recent left temporoparietal ischemic stroke in the territory of the left sylvium which is occluded with an appearance of laminar cortical necrosis (white arrow).
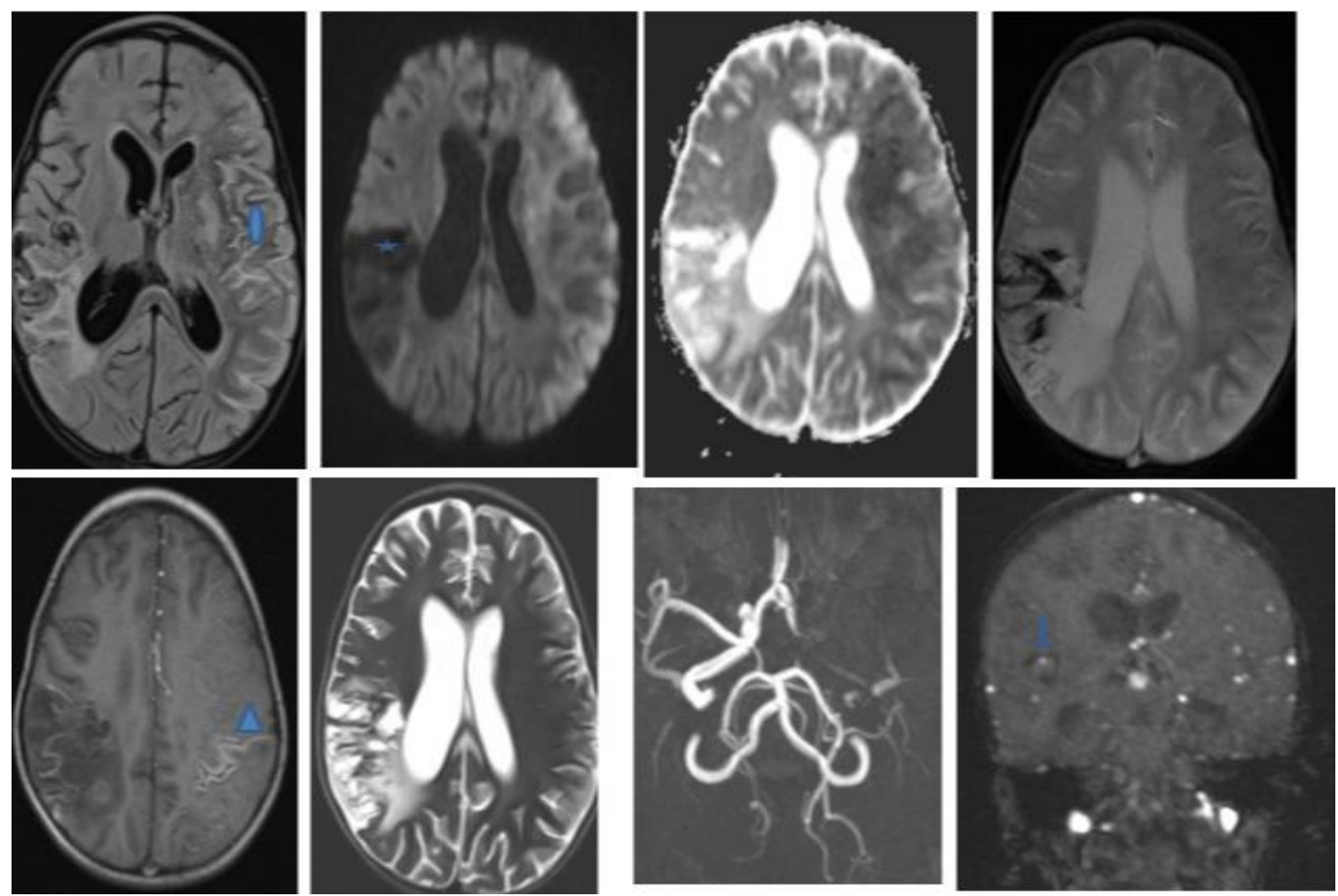

Fig-2: Brain MRI with injection of Gadolinium:

$\star$ Appearance evoking an old ischemic stroke of the right posterior junctional territory with hemorrhagic reorganization.

Aneurysmal dilation of the right M4 with mycotic appearance ICMA.

$\Delta$ Recent left temporoparietal ischemic stroke with an aspect of laminar cortical necrosis. 
Following the worsening of his condition and the development of a left hemiplegia, a control by encephalic MRI was carried out showingmultiple and extensive recent ischemic strokes of the right anterior cerebral and sylvian territories which are occluded associated to petechial hemorrhagic reorganization and microbleeds. It was associated with an onset minimal posterior occipital subarachnoid hemorrhage.

\section{DISCUSSION}

Infectious endocarditis is a rare disease with variable clinical manifestations and is generally difficult to diagnose. It is due to damage of the valvular or adjacent endothelium which is normally nonthrombogenic, thus allowing platelet adhesion and the formation of an initially non-septic thrombus and which, in case of bacteremia, even transient, can give septic vegetations which escape the immune system [3]. Infectious endocarditis can give rise to numerous systemic complications related either to immunological phenomena (immune complexes) or to the migration of septic emboli.

Neurological complications represent a nonnegligible entity of general complications, their incidence varying between $10 \%$ and $35 \%$ depending on the series. Their particularity lies in the fact that they are life-threatening for the patient and therefore can completely change the management and/or the prognosis [4].

They are secondary to the migration of vegetation into the cerebral circulation, which, depending on its septic nature or not, can give different clinical pictures: ischemic embolic vascular accidents, meningitis, cerebral hemorrhages and brain abscesses [5].

As is illustrated in our case, the patient presented on CT a left ischemic stroke of the territory of the left sylvian artery and a mycotic aneurysm of the M3 branch of the right sylvian artery.

Intracranial mycotic aneurysms [ICMA] or infectious aneurysms are rare and represent less than $10 \%$ of the neurological complications of infectious endocarditis $[6,7]$. The septic embolus, localized in the vascular lumen or at the level of the vasa vasorum, creates a suppurative inflammatory lesion of the vascular wall at the level of the intima, which diffuses by contiguity, in and out. Secondary parenchymal ischemic lesions are sometimes observed. The course without treatment is a rupture of the vascular wall, creating a picture of cerebral meningeal hemorrhage. Among the infectious agents capable of causing ICMA, alpha-hemolytic streptococci of the viridans group and Staphylococcus aureus are the pathogens most frequently involved (responsible for $50 \%$ and $10 \%$ of ICMA, respectively) [8]. ICMAs are spindle-shaped, irregular with an ill-defined aneurysm neck. They are classically multiple ( $25 \%$ of cases), located distally and at the bifurcations of the sylvian cerebral artery [9]. The clinical manifestations of unruptured ICMA are variable and nonspecific: fever, headache, convulsions and focal deficit

Symptomatic ischemic strokesare reported in 10 to $35 \%$ of AEs [10], which was the case in our patient; and represents the most frequent neurological complication: approximately $50 \%$ of the neurological complications of AEs are ischemic strokes linked to emboli from vegetations. The clinical consequences depend on the size of the embolus and the area affected. Thus, more than $40 \%$ of embolic events in the central nervous system affect the territory of the middle cerebral artery [11]. Two echocardiographic risk factors for an embolic event have been established: the size greater than $10 \mathrm{~mm}$ and the mobile nature of the vegetations [12]. Three studies have shown that the incidence of brain emboli is more common in left mitral than aortic endocarditis [13].

The discovery of a cerebral hemorrhagic complication requires defining and treating the cause of the bleeding. It can be intra-parenchymal or subarachnoid, and explained by three main mechanisms: hemorrhagic transformation of an ischemic stroke, rupture of an intracranial mycotic aneurysm and rupture of an intracranial vessel by necrotizing arteritis [12]. Brain MRI, via the T2 * sequence, can detect silent microscopic hemorrhagic lesions, "microbleeds", not visible on CT.

\section{Conclusion}

Mycotic aneurysm is a rare complication of infectious endocarditis, its course is unpredictable. The presence of a febrile neurologic symptomatology in a patient with or without a prior valvular problem may be suggestive of an infectious endocarditis, thus allowing early diagnosis and well-conducted treatment and considerably improving the prognosis.

Conflicts of Interest: The authors declare no conflict of interest

Author Contributions: All authors read and approved the final version of the manuscript.

\section{REFERENCES}

1. Grillo P, Velly L, Bruder N. Accident vasculaire cérébral hémorragique: nouveautés sur la prise en charge. Annales Françaisesd'Anesthésie et de Réanimation. 2006;25(8):868-873.

2. Sonneville R, Klein I, Bouadma L, Mourvillier B, Regnier B, Wolff M. Complications neurologiques des endocardites infectieuses. Réanimation. 2009; 18(7):547-555.

3. Delahaye F. Endocardite infectieuse: valvulopathies cardiaques de l'adulte. Pascal and 
Francis Bibliographic Databases. 2000;50(16):1665-1671.

4. Mourvillier B, Trouillet JL, Timsit JF, Baudot J, Chastre J, Régnier B, Gibert C, Wolff M. Infective endocarditis in the intensive care unit: clinical spectrum and prognostic factors in 228 consecutive patients. Intensive care medicine. 2004 Nov;30(11):2046-52.

5. Francioli P, Scheld W, Whiteley R, Durack D. Infections of the central nervous system. Lippincott williamswilkins. 2014.

6. Gillinov AM, Shah RV, Curtis WE, Stuart RS, Cameron DE, Baumgartner WA, Greene PS. Valve replacement in patients with endocarditis and acute neurologic deficit. The Annals of thoracic surgery. 1996 Apr 1;61(4):1125-30.

7. Kannoth S, Thomas SV. Intracranial microbial aneurysm (infectious aneurysm): Current options for diagnosis and management. Neurocrit Care. 2009;11:120-9.

8. Baddour LM, Wilson WR, Bayer AS, Fowler Jr VG, Bolger AF, Levison ME, Ferrieri P, Gerber MA, Tani LY, Gewitz MH, Tong DC. Infective endocarditis: diagnosis, antimicrobial therapy, and management of complications: a statement for healthcare professionals from the Committee on Rheumatic Fever, Endocarditis, and Kawasaki Disease, Council on Cardiovascular Disease in the Young, and the Councils on Clinical Cardiology, Stroke, and Cardiovascular Surgery and Anesthesia, American Heart Association: endorsed by the Infectious Diseases Society of America. Circulation. 2005 Jun 14;111(23):e394-434.

9. Peters PJ, Harrison T, Lennox JL. A dangerous dilemma: management of infectious intracranial aneurysms complicating endocarditis. Lancet Infect Dis. 2006;6:742-8. 